\title{
Anti-sampling-distortion compressive wideband spectrum sensing for Cognitive Radio
}

\author{
Yipeng Liu* and Qun Wan \\ Department of Electronic Engineering, \\ University of Electronic Science and Technology of China (UESTC), \\ Chengdu 611731, China \\ E-mail: liuyipeng@uestc.edu.cn \\ E-mail: wanqun@uestc.edu.cn \\ ${ }^{*}$ Corresponding author
}

\begin{abstract}
In this paper, Compressed Sensing (CS) is introduced to wideband spectrum sensing for cognitive radio to deal with the too high sampling rate challenge. The standard sparse signal recovery of CS does not consider the distortion in the Analogue-to-Information Converter (AIC). Thus we define a new sparse signal model with bounded sampling error, and an Anti-Sampling-Distortion Constraint (ASDC) is deduced. We combine the $\ell_{1}$ norm based sparse constraint with the ASDC to get a novel robust sparse signal recovery operator. Numerical simulations demonstrate that the proposed method outperforms standard sparse wideband spectrum sensing in accuracy, denoising ability, etc.
\end{abstract}

Keywords: cognitive radio; wideband spectrum sensing; CS; compressive sensing; sparse signal recovery; mobile communication; AIC; analogue to information converter; sampling distortion.

Reference to this paper should be made as follows: Liu, Y. and Wan, Q. (2011) 'Anti-sampling-distortion compressive wideband spectrum sensing for Cognitive Radio', Int. J. Mobile Communications, Vol. 9, No. 6, pp.604-618.

Biographical notes: Yipeng Liu received the BEng Degree from University of Electronic Science and Technology of China (UESTC), Chengdu, China, in 2006. Since 2006, he has been a PhD candidate at UESTC. During 2007-2008, he was a Research Assistant at the China Electronic Technology Corporation. Since 2010, he has been a visiting $\mathrm{PhD}$ candidate at Tsinghua University. His general research interest focuses on signal processing for wireless communication.

Qun Wan received the BS Degree from Nanjing University in 1993, the MS Degree from UESTC in 1996, and the PhD Degree from UESTC in 2001. During 2001-2002, he was a post-doctor at Tsinghua University, where he participated in cellular localisation program. In 2003, he was a Technical Staff at UTstarcom. Since 2004, he has been a Professor in the Department of Electronic Engineering at the University of Electronic Science and Technology of China (UESTC). His research interests include sparse and array signal processing, mobile and indoor localisation. He is a Senior Member of CIE. 


\section{Introduction}

Current static spectrum assignment policy leads to a paradox between the overcrowded spectrum and the pervasiveness of idle frequency bands (FCC, 2002). To deal with the problem, a fast developing revolutionary technique named Cognitive Radio (CR) is proposed to achieve more flexible spectrum management policies and technologies for dynamic radio spectrum access (Mitola and Maguire, 1999; Haykin, 2005). CR is defined as a paradigm for wireless communication in which either a network or a wireless node rapidly tunes its transmission or reception parameters to communicate efficiently avoiding interference to and from licensed or unlicensed users. This alteration of parameters is based on the dynamical monitoring of the external and internal radio environment, such as radio frequency spectrum, user behaviour and network state. It is regarded as the next big thing in wireless communication.

Spectrum sensing is an essential function of CR. It autonomously detects the spectrum usage without harmful interference, and then the CR users can be allowed to utilise the unused primary frequency bands. Current spectrum sensing is usually performed in two steps (Ghasemi and Sousa, 2008): the first step, called coarse spectrum sensing, is to detect the Power Spectrum Density (PSD) level of primary bands; and the second step, called feature detection or multi-dimensional sensing (Yucek and Arslan, 2009; Yucek, 2007), is to estimate other signal space allowing accessible for CR, such as Direction Of Arrival (DOA) estimation, spread spectrum code identification, waveform identification, etc.

Coarse spectrum sensing requires fast and accurate power spectrum detection over a wideband and even Ultra-Wideband (UWB) for many CR missions (Cabric et al., 2006). One approach utilises a bank of tunable narrowband bandpass filters to search one frequency band at a time. However, it requires an enormous number of RF components, which leads to high cost and much time-consuming. The other one is a wideband circuit using a single RF chain followed by high-speed DSP to flexibly search over multiple frequency bands concurrently (Sahai and Cabric, 2005). High sampling rate requirement and the resulting large number of data for processing are the major problems (Tian and Giannakis, 2007).

Compressive Sensing (CS) is a very promising technique to deal with these problems (Laska et al., 2006, 2007; Donoho, 2006; Candés et al., 2006). CS is a new signal processing technique that can reconstruct the signal with a much fewer randomised samples than Nyquist sampling with high probability on condition that the signal has a sparse representation. The sampling measurement matrix should be incoherent with the dictionary. Orthogonal Matched Pursuit (OMP) (Needell and Vershynin, 2009), Least-Absolute Shrinkage and Selection Operator (LASSO) (Chen et al., 1999) and Dantzig Selector (DS) (Candes and Tao, 2007) are the major ways to recovering the sparse signal.

The standard sparse signal recovery algorithms, such as BP, were used to recover the spectrum from the random samples obtained by Analogue to Information Converter (AIC) (Laska et al., 2006, 2007) as Figure 1 shows. In Tian and Giannakis (2007), an analogue-to-digital converter has been used to transform the analogue received signal into a digital signal by sampling at the Nyquist rate. Next, sub-Nyquist random sampling is applied to the sampled vector to compress it into a shorter vector and then the power spectrum is reconstructed by solving the BP problem. The received analogue signal 
can be sampled at the information rate of the signal using an AIC (Laska et al., 2006, 2007). Here, the CS is embedded in the AIC. The same sparse signal recovery methods can be used to estimate the original PSD. In Tian and Giannakis (2007), wavelet edge detection has been used to detect the spectrum borders in the estimated spectrum and the detection's performance has been evaluated in terms of mean square error through simulations.

Figure 1 The proposed compressive wideband spectrum sensing structure (see online version for colours)

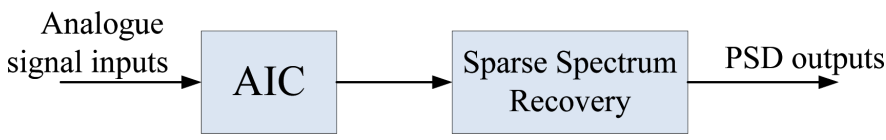

However, traditional methods do not consider uncertainties in AIC. In fact, sampling distortion can be resulted from various types of effects, such as aliasing, aperture effect, jitter or deviation from the precise sample timing intervals, noise, slew rate limit error, and other non-linear effects (Oppenheim and Shaffer, 1989). Sampling distortion would change the compressive sampling signal model and make the present sparse signal recovery methods mismatch the signal. Thus, the performance of the CWSS would be deteriorated.

To prevent performance degradation caused by sampling distortion, this paper proposes a robust compressive wideband spectrum sensing method. Sampling distortion is modelled as a bounded additive error. The estimated signal approximates the sparse signal with a mixed $\ell_{1}$ and $\ell_{2}$ norms with respect to the sampling distortion parameter. Then we combine the sparse constraint with the mixed $\ell_{1}$ and $\ell_{2}$ norms constraint to get the robust compressive wideband spectrum sensing operator. Numerical simulations demonstrate that the proposed operator has a better reconstructing performance with the sampling distortion than traditional methods.

In the rest of the paper, the CS theory is reviewed briefly in Section 2. Section 3 gives the signal model. Section 4 provides a typical representative of the traditional compressive wideband spectrum sensing method; In Section 5, the proposed robust wideband spectrum sensing against sampling distortion is given. In Section 6, performance enhancement of the proposed operator is demonstrated by numerical experiments. Finally, Section 7 draws conclusion and discusses on further work.

\section{Compressed sensing review}

Sparsity widely exists in wireless signals (Candes and Wakin, 2008). Considering a signal $\mathbf{x}$ which can be expanded by an orthogonal complete dictionary with the representation given as

$$
\mathbf{x}_{N \times 1}=\boldsymbol{\Psi}_{N \times N} \mathbf{b}_{N \times 1},
$$

when most elements of the vector $\mathbf{b}$ are zeros, the signal $\mathbf{x}$ is sparse. If the number of nonzero elements of $\mathbf{b}$ is $S(S<<N)$, the signal is said to be $S$-sparse. CS provides an alternative to the well-known Shannon sampling theory. On condition that the signal is 
sparse, CS performs non-adaptive measurement of the informative part of the signal directly.

In CS, instead of measure the signal directly by Nyquist sampling, a random measurement matrix $\boldsymbol{\Phi}$ is used to sample the signal. In matrix notation, the obtained random sample vector can be represented as:

$$
\mathbf{y}_{M \times 1}=\boldsymbol{\Phi}_{M \times N} \mathbf{x}_{N \times 1} .
$$

The measurement matrix should satisfy the Restricted Isometry Property (RIP) which provides a guarantee on the performance of $\boldsymbol{\Phi}$ in CS. It can be stated as Donoho (2006), Candés et al. (2006) and Candes and Wakin (2008):

$$
\left(1-\delta_{s}\right)\|\mathbf{y}\|_{2}^{2} \leq\|\mathbf{\Phi} \mathbf{y}\|_{2}^{2} \leq\left(1+\delta_{s}\right)\|\mathbf{y}\|_{2}^{2},
$$

for all the $S$-sparse $\mathbf{y}$. In equation (3), $\|\mathbf{y}\|_{2}=\left(\sum_{i}\left|y_{i}\right|^{2}\right)^{1 / 2}$ is the $\ell_{2}$ norm of the vector $\mathbf{y}=\left[y_{1}, y_{2}, \ldots, y_{M}\right]^{T}$. The RIP $\delta_{s} \in(0,1)$ is defined as the smallest constant for which this property holds for all $S$-sparse vectors $\mathbf{y}$.

There are three kinds of frequently used measurement matrices:

- Non-Uniform Sub-sampling (NUS) or Random Sub-sampling matrices which are generated by choosing $M$ separate rows uniformly at random from the unit matrix $\mathbf{I}_{N}$

- Matrices formed by sampling the i.i.d. entries $(\boldsymbol{\Phi})_{\mathrm{ij}}$ from a white Gaussian distribution

- Matrices formed by sampling the i.i.d. entries $(\boldsymbol{\Phi})_{\mathrm{ij}}$ from a symmetric Bernoulli distribution and the elements are $\pm 1 / \sqrt{N}$ with probability $1 / 2$ each.

The hardware performing random samplings of type 1 and 3 can refer to Laska et al. (2006, 2007). When the RIP holds, a series of recovering algorithm can reconstruct the sparse signal. One is greedy algorithm, such as Matched Pursuit (MP), OMP; another group is convex program, such as Basis Pursuit (BP), LASSO and DS. In these algorithms, DS has almost the same performance as LASSO. Convex program algorithm has a more reconstruction accuracy while greedy algorithm has less computational complexity.

\section{Signal model}

According to the measured data (FCC, 2002; Tian and Giannakis, 2007), we may suppose that the receiver provides a wideband monitoring frequency range and the received signal, $y(t), 0 \leq t \leq T$, just occupies a small parts of non-consecutive frequency bands with different PSD levels. It can be represented as

$$
y(t)=\sum_{i=1}^{N} c_{i} \psi_{i}(t),
$$

where $\boldsymbol{\Psi}_{i}(t)$, which is the Fourier basis functions and $c_{\mathrm{i}}$ is the weighting factors indicating the PSD. In the weighting vector $\mathbf{c}=\left[c_{1}, c_{2}, \ldots, c_{N}\right]^{T}$, most of the elements are zeros or trivial. 


\subsection{Traditional sparse spectrum model}

In traditional Nyquist sampling, the time window for sensing is $t \in\left[0, N T_{0}\right] . N$ samples are needed to recover the PSD $r$ without aliasing, where $T_{0}$ is the Nyquist sampling duration. A digital receiver converts the continuous signal $y(t)$ to a discrete complex sequence $\boldsymbol{y}$ of length $M$. For illustration convenience, we detail the algorithm in discrete setting as it did in Haykin (2005), Zeng et al. (2011), Tian et al. (2008), Tian (2008), Elsner et al. (2009), Wang et al. (2009) and Polo et al. (2009):

$$
\mathbf{y}=\Psi_{\mathbf{y}_{\mathrm{t}}} \mathbf{y}_{\mathrm{t}} \in \mathbf{R}^{N},
$$

where $\mathbf{y}_{t}$ represents an $N \times 1$ vector with elements $\boldsymbol{y}_{t}[n]=x(t), t=n T_{0}, n=1, \ldots, N$, and $\boldsymbol{S}$ is an $M \times N$ projection matrix. For example, when $\Psi=\mathbf{F}_{N}$, model (5) amounts to frequency domain sampling, where $\mathbf{F}_{N}$ is the $N$-point unitary Discrete Fourier Transform (DFT) matrix. Given the sample set $\boldsymbol{y}$ when $M<N$, compressive spectrum sensing can reconstruct the spectrum with reduced amount of sampling data.

Processing $y(t)$ in such a broadband needs high sampling rate which has high cost. Besides, too many sampling measurements inevitably increase computational burden for digital signal processors. Nevertheless, spectrum sensing requires a fast and accurate algorithm. Considering $\boldsymbol{y}_{t}$ has a sparse representation in frequency domain, we use an $M \times N$ random projection matrix $\boldsymbol{S}_{c}$ to sample signals as the type 1 random measurement in Section 2, i.e. $\boldsymbol{y}=\boldsymbol{S}_{c} \boldsymbol{y}_{t}$, where $M<N$.

In practice, the analogue baseband signal $y(t)$ is sampled using an Analogue-to-Information Converter (AIC). The AIC can be conceptually modelled as an ADC operating at Nyquist rate, followed by CS operation. Then $\boldsymbol{y}$ is obtained directly from continuous time signal $y(t)$ by AIC (Laska et al., 2006, 2007). Here we incorporate the AIC to the wideband spectrum sensing architecture as Figure 1.

\subsection{Practical sparse spectrum model with sampling distortion}

The AIC is a non-ideal device with various physical limitations. Various types of effects can lead to sampling distortions, including:

1 Aliasing. The ideal sampling theorem assumes that the signal is bandlimited.

However, in practice, bandlimitated signal cannot be guaranteed for the time-limited signal. Since the sampled continuous signals are almost time-limited (e.g., at most spanning the lifetime of the sampling device in question), it gives out that they cannot be bandlimited, according to the time-frequency relationship. Thus, aliasing would happen and lead to sampling distortions.

2 Integration effect or aperture effect. This effect results from the fact that the sample is obtained as time average within a sampling region, rather than just being equal to the signal value at the sampling instant. The integration effect is readily noticeable in photography when the exposure is too long and creates a blur in the image. An ideal camera would have an exposure time of zero. In a capacitor-based sample and hold circuit, the integration effect is introduced because the capacitor cannot instantly change voltage thus requiring the sample to have non-zero width.

3 Jitter or deviation from accurate sampling time intervals due to limitation of the physical device. 
4 Noise, including thermal sensor noise, analogue circuit noise, etc.

5 Slew rate limit error. It can be caused by inability when the device's output changes sufficiently fast.

6 Quantisation as a consequence of the finite precision of words that represent the converted values. In practice, the measurement matrix can only be able to incorporate finite frequencies. The estimated spectrum can only be represented with respect to the finite discrete frequencies. However, the primary signal's spectrum can be in any position within the sampling frequency range. When the active primary signals are not located in the divided frequencies, quantisation error would occur and lead to sampling distortion. It is more serious when the sampling frequency band is wide.

7 Error due to other non-linear effects when converting input voltage to output value can also result in sampling error.

All the above effects can give birth to sampling distortion in AIC, but the traditional sparse spectrum model (5) does not consider sampling distortion. Performance degrade would be caused by the inaccurate signal model. To make the sparse spectrum model more practical, the sampling distortion can be modelled as a bounded additive noise on the measurement matrix. The new sparse spectrum model with sampling distortion can be formulated as:

$$
\begin{aligned}
& \mathbf{y}=\Psi \mathbf{y}_{t}=\Psi \Phi \mathbf{b}=\mathbf{A b}, \quad \mathbf{b} \in \mathbf{R}^{N}, \\
& \mathbf{B}=\mathbf{A}+\mathbf{V},
\end{aligned}
$$

where $M \times 1$ vector $\mathbf{y}$ is the random samples, the real $M \times N$ matrix $\mathbf{A}$ is unknown, $\mathbf{B}$ is the $M \times N$ observed matrix with an additive noise $\mathbf{V}$, and the $N \times 1$ vector $\mathbf{b}$ is sparse with most of its elements equal zero or close to zero.

Without loss of generality, we assume that $\mathbf{V}$ is deterministic and satisfies:

$$
\|\mathbf{V}\|_{\infty} \leq \delta
$$

where $\delta>0$, and $\|\mathbf{V}\|_{\propto}$ stands for the $\ell_{\propto}$ norm for the matrix $\mathbf{V}$, which gives the maximum of $\sum_{i=1}^{N}\left|v_{i, j}\right|$ for all $j$. The $v_{i, j}$ is the element of matrix $\mathbf{V}$ in $i$ th column and $j$ th row. The parameter $\delta$ gives a restriction on the bound of the observed measurement matrix noise $\mathbf{V}$.

If $\mathbf{V}$ is random, condition (8) can also be guaranteed with a probability close to 1; and the values of $\delta$ can be indicated correspondingly. Thus, the result that we prove for deterministic $\mathbf{V}$ is extended in a trivial way to their random version satisfying this assumption.

\section{Basis Pursuit based compressive wideband spectrum sensing}

CS theory asserts that, if a signal has sparse representation in a certain space, one can use the random measurement to obtain the samples and reconstruct it with overwhelming probability by optimisation techniques, as stated in Section 2. The required random samples for recovery are far fewer than Nyquist sampling requires. 
To find the unoccupied spectrum for the secondary access, the signal in the monitoring band is down-converted to baseband and sampling the resulting analogue signal through an AIC that produces samples at a rate below the Nyquist rate.

Now we estimate the frequency response of $y(t)$ from the measurement $\mathbf{y}$ based on the transformation equality $\mathbf{y}=\mathbf{S}_{c} \mathbf{F}_{N}^{-1} \mathbf{r}$, where $\mathbf{r}$ is the $N \times 1$ frequency response vector of signal $y(t), \boldsymbol{F}_{N}$ is the $N \times N$ Fourier transform matrix, and $\mathbf{S}_{c}$ is the $M \times N$ matrix which is obtained by randomising the column indices and getting the first $M$ columns. The real measurement matrix can be formulated as:

$$
\mathbf{A}=\mathbf{S}_{c} \mathbf{F}_{N}^{-1} .
$$

In practice, as it states in Section 3, the sampling may suffer various physical effects and get the measurement matrix distortion as equation (7), and the obtained observed measurement matrix should be

$$
\mathbf{B}=\mathbf{S}_{c} \mathbf{F}_{N}^{-1}+\mathbf{V} .
$$

With the observed measurement matrix $\mathbf{B}$ and the random measurement vector $\mathbf{y}$, the CWSS can be formulated as:

$$
\begin{aligned}
& \hat{\mathbf{r}}=\underset{\mathbf{r}}{\arg \min }\|\mathbf{r}\|_{0}, \\
& \text { s.t. } \mathbf{y}=\mathbf{B r}
\end{aligned}
$$

where $\|\mathbf{r}\|_{0}$ is the $\ell_{0}$ norm which counts the number of nonzero entries of the vector $\mathbf{r}$. The minimisation of the $\ell_{0}$ norm is the optimal to describe the sparse distribution. However, it is not a convex function and the formulated CWSS (11) (12) is not a convex programming.

To convert the CWSS model to a convex programming, the $\ell_{0}$ norm is relaxing to the $\ell_{1}$ norm, and the BP is used to performance CWSS. The globe optimal sparse solution can be obtained. The BP based CWSS (BP-CWSS) can be formulated as (Tian and Giannakis, 2007; Chen et al., 1999):

$$
\begin{aligned}
& \hat{\mathbf{r}}=\underset{\mathbf{r}}{\arg \min }\|\mathbf{r}\|_{1}, \\
& \text { s.t. } \mathbf{y}=\mathbf{B r},
\end{aligned}
$$

where $\|\mathbf{r}\|_{1}=\sum_{i}\left|r_{i}\right|$ is the $\ell_{1}$ norm of the vector $\mathbf{r}=\left[r_{1}, r_{2}, \ldots, r_{N}\right]^{T}$. This problem is a Second Order Cone Program (SOCP) and thereby can be solved efficiently using standard software packages. It finds the smallest L1 norm of coefficients among all the decompositions that the signal is decomposed into a superposition of dictionary elements. It is a decomposition principle based on a true global optimisation.

The standard BP does not allow sampling noise. In contrast, LASSO, also called Basis Pursuit Denoising (BPDN), has additional advantage of noise suppression (Tibshirani, 1996). It is a shrinkage and selection method for linear regression. It minimises the usual sum of squared errors, with a bound on the sum of the absolute values of the coefficients. To get more accuracy, we can reformulate the CWSS based on LASSO as 


$$
\begin{gathered}
\hat{\mathbf{r}}=\underset{\mathbf{r}}{\arg \min }\|\mathbf{r}\|_{1}, \\
\text { s.t. }\|\mathrm{Br}-y\|_{2} \geq \mu,
\end{gathered}
$$

where $\mu$ is a parameter bounding the amount of noise in the data. As it directly uses the sparsity to recover the wide frequency band spectrum by LASSO with reduced measurements, we named it as LASSO based compressive wideband spectrum sensing (LASSO-CWSS). The problem of LASSO is a quadratic programming problem or more generally, a convex optimisation problem, which can be tackled by standard numerical analysis algorithms. The solution for it has been well investigated (Tibshirani, 1996; Efron et al., 2004; Kim et al., 2007a, 2007b). A number of convex optimisation software, such as cvx (Boyd and Vandenberghe, 2004), SeDuMi (Sturm, 1999) Yalmip (Lofberg, 2006), can be used to solve the problem.

\section{Robust compressive wideband spectrum sensing with sampling distortion}

Traditional ways for CWSS are designed for the sparse signal model (5). It formulates with respect to the real measurement matrix without any sampling distortion. However, in practice the continuous signal is sampled using the AIC, which is a non-ideal device with various physical effects leading to the deviations from the theoretically perfect reconstruction capabilities. Conditions (14) and (16) would mismatch the more practical sparse spectrum signal model (6) and (7). The sampling distortion would lead to the performance degeneration of the BP-CWSS and the LASSO-CWSS.

In cognitive radio, the spectrum sensing accuracy is vitally important. The inaccurate wideband spectrum sensing would lead to missed detection or false detection. The missed detection can make the cognitive radio occupy the spectrum, which the active primary users are using. Thus, both the cognitive radio and the primary user can cause and get interference from each other. Both of their communication quality can seriously degrade. On the other hand, false detection would exclude the cognitive radio to take the spectrum where no primary user is using. As both the cognitive radio and primary user are not taking the spectrum, the basic ideal that using cognitive radio to access the unused spectrum is not realised. Spectrum waste would still exist.

To mitigate the performance degeneration caused by the sampling distortion, we incorporate the sampling distortion information in the sparse wideband spectrum recovery algorithm. Taking advantage of the sparse spectrum signal model (6) (7), we can make up the performance degeneration caused by the sampling distortion in sparse spectrum reconstruction algorithm. To get the robust sparse wideband spectrum estimation algorithm, we can reformulate the square estimation error as:

$$
\begin{aligned}
\|\mathbf{y}-\mathbf{B} \boldsymbol{\theta}\|_{2} & =\|\mathbf{y}-(\mathbf{A}+\mathbf{V}) \mathbf{r}\|_{2} \\
& =\|\mathbf{A r}-(\mathbf{A}+\mathbf{V}) \mathbf{r}\|_{2} \\
& =\|\mathbf{V} \mathbf{r}\|_{2} .
\end{aligned}
$$

Here we define the row vector $\mathbf{v}_{i}, i=1,2, \ldots, M$ of the matrix $\mathbf{V}$ as: 


$$
\mathbf{V}=\left[\begin{array}{c}
\mathbf{v}_{1} \\
\mathbf{v}_{2} \\
\vdots \\
\mathbf{v}_{M}
\end{array}\right] .
$$

Then equation (17) can be reformulated as:

$$
\|\mathbf{V r}\|_{2}=\sqrt{\sum_{m=1}^{M}\left|\mathbf{v}_{m} \mathbf{r}\right|^{2}}
$$

where $|\bullet|$ means the modulus operator of a scalar. It is easy to prove that

$$
\begin{aligned}
\left|\mathbf{v}_{m} \mathbf{r}\right| & =\left|v_{m, 1} r_{1}\right|+\left|v_{m, 2} r_{2}\right|+\cdots+\left|v_{m, N} r_{N}\right| \\
& \leq\left(\left|v_{m, 1}\right|+\left|v_{m, 2}\right|+\cdots+\left|v_{m, N}\right|\right)\left(\left|r_{1}\right|+\left|r_{2}\right|+\cdots+r_{N}\right), \\
& =\left\|\mathbf{v}_{m}\right\|_{1}\|\mathbf{r}\|_{1}
\end{aligned}
$$

where $v_{m, i}, i=1,2, \ldots, N$, is the $i$ th element of the vector $\mathbf{v}_{m}$; and $r_{i}, i=1,2, \ldots, N$, is the $i$ th element of the vector $\mathbf{r}$. Taking equation (20) into equation (19), we can get

$$
\|\mathbf{V r}\|_{2} \leq \sqrt{\sum_{m=1}^{M}\left(\left\|\mathbf{v}_{m}\right\|_{1}\|\mathbf{r}\|_{1}\right)^{2}}
$$

Then, obviously we have

$$
\begin{aligned}
\|\mathbf{V} \mathbf{r}\|_{2} & \leq \sqrt{\sum_{m=1}^{M}\left(\|\mathbf{v}\|_{1, \text { max }}\|\mathbf{r}\|_{1}\right)^{2}} \\
& =\sqrt{M\left(\|\mathbf{v}\|_{1, \text { max }}\|\mathbf{r}\|_{1}\right)^{2}}, \\
& =\sqrt{M}\|\mathbf{V}\|_{\infty}\|\mathbf{r}\|_{1}
\end{aligned}
$$

where $\|\mathbf{v}\|_{1, \max }$ means the maximum one of all the values of the $\left\|\mathbf{v}_{i}\right\|_{1}, i=1,2, \ldots, M$. With the condition (8), we can get:

$$
\|\mathbf{V r}\|_{2} \leq \sqrt{M} \delta\|\mathbf{r}\|_{1}
$$

Combining equations (17) and (23), we get the Anti-Sampling-Distortion Constraint (ASDC), it can be formulated as:

$$
\|\mathbf{y}-\mathbf{B r}\|_{2} \leq \sqrt{M} \delta\|\mathbf{r}\|_{1} .
$$

It is a relaxing version of the standard square error bound constraint and gives the relationship between the square error and sparsity measure of the estimated signal with the sampling distortion parameter incorporated.

Combining the ASDC with the sparse constraint, we get the anti-sampling-distortion compressive wideband spectrum sensing (ASD-CWSS) as:

$$
\begin{gathered}
\min \|\mathbf{r}\|_{1} \\
\text { s.t. }\|\mathbf{y}-\mathbf{B r}\|_{2} \leq \sqrt{M} \delta\|\mathbf{r}\|_{1}
\end{gathered}
$$


It can be reformulated as

$$
\begin{gathered}
\min (t) \\
\text { s.t. }\|\mathbf{y}-\mathbf{B r}\|_{2} \leq \sqrt{M} \delta t . \\
\|\mathbf{r}\|_{1}<t
\end{gathered}
$$

Obviously, equation (26) is a convex programming and can be solved by a host of numerical methods in polynomial time. Similar to the solution of LASSO-CWSS, the optimal $\mathbf{r}$ of ASD-CWSS can also be obtained efficiently using some convex programming software packages. Such as cvx (Boyd and Vandenberghe, 2004), SeDuMi (Sturm, 1999); Yalmip (Lofberg, 2006), etc.

\section{Simulation results}

Numerical experiments are presented to illustrate performance of the proposed ASDC-CWSS for cognitive radio. Here we consider a base band signal with its frequency range from $0 \mathrm{~Hz}$ to $500 \mathrm{MHz}$. Among the whole monitoring band, the noise level is set to be in random Gaussian distribution between 0 and 10. Four primary signals are located at 30-70 MHz, $120-180 \mathrm{MHz}, 300-340 \mathrm{MHz}, 420-460 \mathrm{MHz}$. And their corresponding normalised PSD levels fluctuate in the range of 100-140, 70-110, 130-170, and 110-150. The primary signals with random phase are contaminated by a zero-mean Additive Gaussian White Noise (AGWN) which makes the Signal Noise Ratio (SNR) be $13 \mathrm{~dB}$. Then the wideband spectrum in normalised as Figure 2 shows. The observed measurement matrix noise $\mathbf{V}$ is a complex Gaussian random matrix with the bound of the modulus of elements being $\delta=0.7$.

Figure 2 The spectrum of noiseless active primary signals in the monitoring band

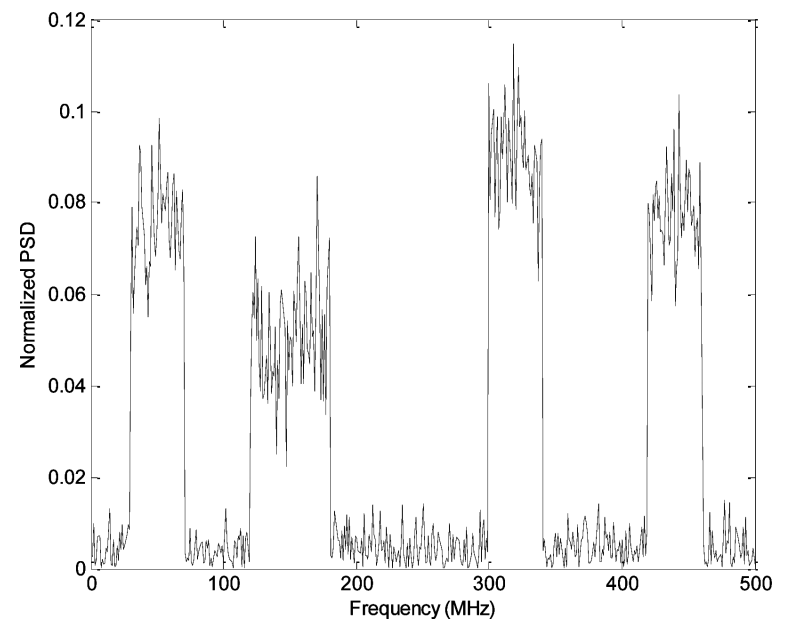

Here we take the contaminated signal as the received signal $y(t)$. As CS theory suggests, we sample $y(t)$ randomly at half the amount of Nyquist sampling. The resulted sub-sample vector by the proposed structure as Figure 1 demonstrated is denoted as $\mathbf{y}$. To make contrast, with the same number of samples, power spectrums with different 
methods are given out in Figures 3 and 4. Figure 3 shows the spectrum estimation result by LASSO-CWSS where $\mu$ is chosen to be $0.1\|\mathbf{y}\|_{2}$ with 1000 trials averaged. Figure 4 shows the result of the proposed ASD-CWSS also with 1000 trials averaged. Figures 3 and 4 show that the ASD-CWSS gives a better reconstruction performance with the same amount of samples. In detail, it is shown that there are too many fake spectrum points in the sub-bands in the absence of active primary signals in Figure 3 for the LASSO-CWSS. The noise levels from LASSO are quite high in the whole monitoring band. However, for the ASD-CWSS, the four occupied bands clearly show up. The noise levels in the inactive bands are quite low; and the variation of the PSD levels in the boundaries of estimated spectrum are quite abrupt and correctly in accordance with the generated sparse spectrum, which would enhance the wavelet edge detection performance much (Tian and Giannakis, 2006). Therefore, the proposed ASD-CWSS outperforms the LASSO-CWSS with a much more accurate spectrum reconstruction as shown in Figures 3 and 4.

Figure 3 The compressive wideband spectrum estimation by the LASSO-CWSS

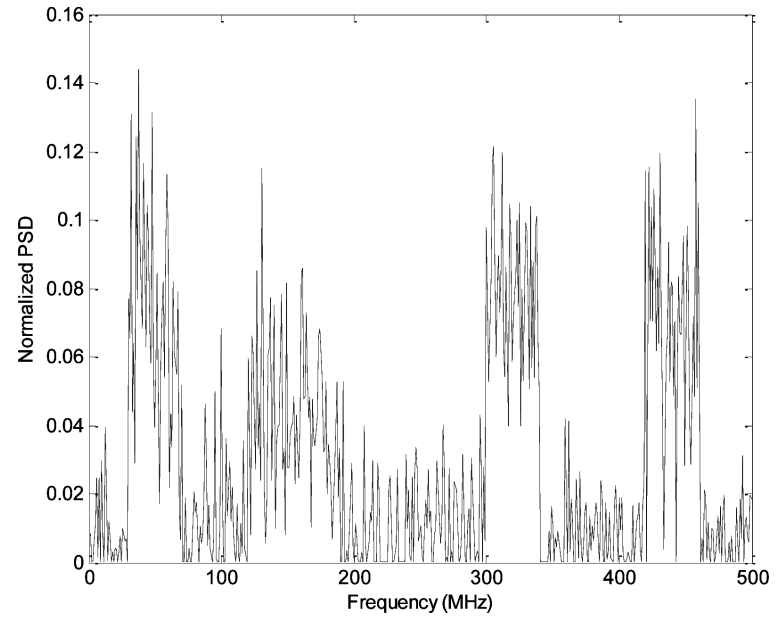

Figure 4 The compressive wideband spectrum estimation by the proposed ASD-CWSS

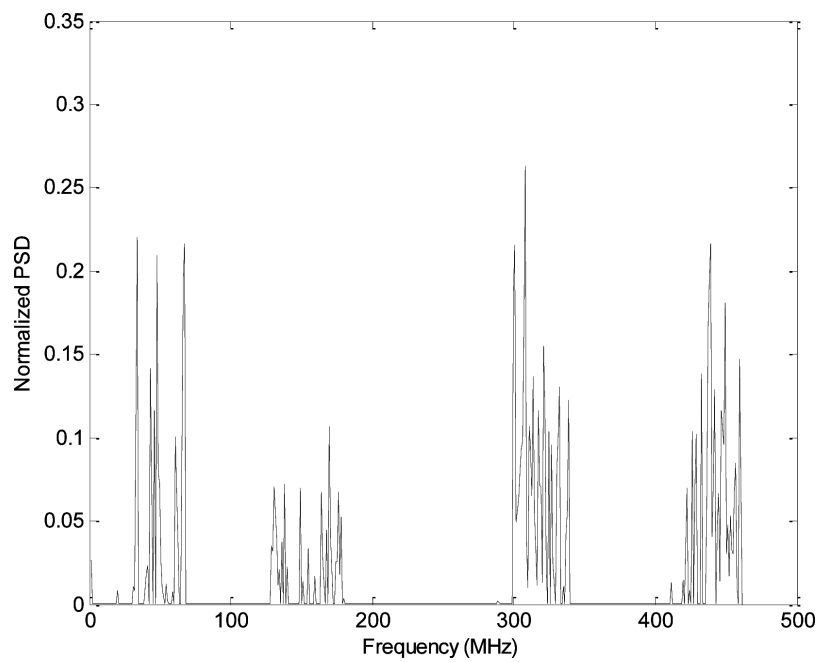


Apart from the edge detection, energy detection is the most popular spectrum sensing approach for cognitive radio. To test compressive wideband spectrum sensing by energy detection, 1000 Monte Carlo simulations are conducted with same parameters given in the last simulation to show the results of average energy in each section of the divided spectrum vector. Here the simulated monitoring band is divided into nine sections as Figure 2. The total energy with each CWSS method is normalised. Table 1 presents the average energy in each sub-band with the corresponding CWSS methods. For the ASD-CWSS, it is obvious that the estimated noise energy of inactive bands is much smaller that the LASSO-CWSS. To quantify the ASD-CWSS's performance gain against LASSO-CWSS, after normalising the total energy with each method, we define the Energy Enhancement Ratio (EER) for the $k$ th sub-band as:

$$
\operatorname{EER}(k)= \begin{cases}\frac{E_{k}^{N}-E_{k}^{S}}{E_{k}^{S}}, & \text { for active subbands } \\ \frac{E_{k}^{S}-E_{k}^{N}}{E_{k}^{S}}, & \text { for inactive subbands }\end{cases}
$$

where $E_{k}^{N}$ represents the energy in the $k$ th sub-band range obtained by the newly proposed ASD-CWSS, and $E_{k}^{S}$ represents the energy vector in the $k$ th sub-band range obtained by the LASSO-CWSS.

Table 1 The total energy in each subband with two compressive wideband spectrum sensing methods and the EBR

\begin{tabular}{lccccccccc}
\hline & 1 & 2 & 3 & 4 & 5 & 6 & 7 & 8 & 9 \\
\hline LASSO-CWSS & 0.0298 & 0.1601 & 0.0335 & 0.1854 & 0.0985 & 0.2052 & 0.0685 & 0.1836 & 0.0352 \\
ASD-CWSS & 0.0036 & 0.2918 & 0.0000 & 0.0990 & 0.0002 & 0.3552 & 0.0013 & 0.2484 & 0.0006 \\
EER & 0.8792 & 0.8222 & 1.0000 & 0.4662 & 0.9979 & 0.7307 & 0.9811 & 0.3524 & 0.9830 \\
\hline
\end{tabular}

This performance function can quantify how much energy increased to enhance the probability of correct energy detection of the active primary bands and how much denoising performance is enhanced. The EER in Table 1 clearly tells the how great the improvement achieved by the proposed ASD-CWSS method. Although there is a sub-band with declined energy, the other active sub-bands have a large energy enhancement and the denoising performance in inactive sub-bands is improved much.

By the way, in 1000 Monte Carlo simulations, on our hardware condition, the average computing periods for the LASSO-CWSS and the ASD-CWSS are $71.7342 \mathrm{~s}, 82.3906 \mathrm{~s}$ respectively. The latter way has a little longer computing period than that of the former one, but the ASD-CWSS gets considerable enhancement of spectrum estimation accuracy with the same number of samples, which qualifies it as an more suitable candidate for wideband spectrum sensing.

\section{Conclusion}

Spectrum scarsity is a big problem for mobile communication. Cognitive radio is the most promising way to deal with it. Wideband spectrum sensing is an essential 
functionality for cognitive radio to detect the secondary spectrum access opportunity without any harmful interference to the primary user. Too high sampling requirement is the most difficult problem for the robust and fast wideband spectrum sensing. Exploiting the inherent sparsity in the wide monitoring band for cognitive radio, CS based sparse signal estimation can perform wideband spectrum sensing with sub-Nyquist random samples which is obtained by AIC. However, traditional sparse signal recovery algorithms do not consider sampling distortion in AIC. Here we model the sampling error to be a bounded additive noise. To get the robust CWSS against sampling distortion, the standard square error bound constraint is replaced by an ASDC in sparse signal recovery algorithm LASSO. The proposed ASDC is in terms of $\ell_{1}$ and $\ell_{2}$ norms of the estimated spectrum and the error bound in sampling distortion. It is a relaxing version of the standard square error bound constraint and gives the relationship between the square error and sparsity of the estimated signal with the sampling distortion parameter incorporated. Numerical experiments show that the proposed ASD-CWSS has performance superior to the LASSO-CWSS in accuracy and denoising capacity. The proposed ASD-CWSS way can be a more suitable candidate for wideband spectrum sensing.

In the future, compressive wideband spectrum sensing can be introduced to the cognitive radio for muti-antenna system and wireless sensor networks (Daoud and Alani, 2009; Mal-Sarkar et al., 2009). Besides, some more a prior information of spectrum distribution can be incorporated to enhance the spectrum sensing accuracy. Greedy algorithm can also be used to solve the ASD-CWSS problem to get a faster spectrum sensing.

\section{Acknowledgement}

The authors would like to thank Dr. Ying Zhang who performs carefully words and grammar checking for the whole paper. This work was supported in part by the National Natural Science Foundation of China under grant 60772146, the National High Technology Research and Development Program of China (863 Program) under grant 2008AA12Z306 and in part by Science Foundation of Ministry of Education of China under grant 109139 .

\section{References}

Boyd, S. and Vandenberghe, L. (2004) Convex Optimization, Cambridge University Press, New York, NY.

Cabric, D., O’Donnell, I.D., Chen, M.S. and Brodersen, R.W. (2006) 'Spectrum sharing radios', IEEE Circuits and Systems Magazine, Vol. 6, No. 2, pp.30-45.

Candes, E.J. and Tao, T. (2007) 'The Dantzig selector: statistical estimation when $\mathrm{p}$ is much larger than n', Annals of Statistics, Vol. 35, No. 6, pp.2313-2351.

Candes, E.J. and Wakin, M.B. (2008) 'An introduction to compressive sampling', IEEE Signal Processing Magazine, Vol. 25, No. 2, pp.21-30.

Candés, E.J., Romberg, J. and Tao T. (2006) 'Robust uncertainty principles: exact signal reconstruction from highly incomplete frequency information', IEEE Transactions on Information Theory, Vol. 52, No. 2, pp.489-509.

Chen, S.S., Donoho, D.L. and Saunders, M.A. (1999) 'Atomic decomposition by basis pursuit', SIAM Journal on Scientific Computing, Vol. 20, No. 1, pp.33-61. 
Daoud, O. and Alani O. (2009) 'Peak-to-average power ratio reduction technique for MIMO/OFDM systems', International Journal of Mobile Communications, Vol. 7, No. 6, pp.632-645.

Donoho, D. (2006) 'Compressed sensing', IEEE Transactions on Information Theory, Vol. 52, No. 4, pp.1289-1306.

Efron, B., Johnstone, I., Hastie, T. and Tibshirani, R. (2004) 'Least angle regression', Annals of Statistics, Vol. 32, No. 2, pp.407-499.

Elsner, J.P., Braun, M., Jakel, H. and Jondral, F.K. (2009) 'Compressed spectrum estimation for cognitive radios', Proceedings of 19th Virginia Tech Symposium on Wireless Communications, Blacksburg, pp.1-4.

FCC (2002) Spectrum Policy Task Force Report, ET Docket no.02-135, November, USA.

Ghasemi, A. and Sousa, E.S. (2008) 'Spectrum sensing in cognitive radio networks: requirements, challenges and design trade-offs', IEEE Communications Magazine, Vol. 46, No. 4, pp.32-39.

Haykin, S. (2005) 'Cognitive radio: brain-empowered wireless communications', IEEE Journal on Selected Areas of Communications, Vol. 23, No. 2, pp.201-220.

Kim, S.J., Koh, K., Lustig, M., Boyd, S. and Gorinevsky, D. (2007a) 'An interior-point method for large-scale l1-regularized least squares', IEEE Journal on Selected Topics in Signal Processing, Vol. 1, No. 4, pp.606-617.

Kim, S.J., Koh, K., Lustig, M., Boyd, S. and Gorinevsky, D. (2007b) 'An interior-point method for large-scale 11-regularized least squares', Proceedings International Conference on Image Processing (ICIP), Vol. 3, pp.III-117-III-120.

Laska, J., Kirolos, S., Duarte, M.F., Ragheb, T.S., Baraniuk, R.G. and Messoud, Y. (2007) 'Theory and implementation of an analog-to-information converter using random demodulation', IEEE International Symposium on Circuits and Systems (ISCAS), New Orleans, Louisiana, USA, pp.1959-1962.

Laska, J.N., Kirolos, S., Massoud, Y., Baraniuk, R., Gilbert, A., Iwen, M. and Strauss, M. (2006) 'Random sampling for analog-to-information conversion of wideband signals', IEEE Dallas Circuits and Systems Workshop (DCAS), Vol. 1, The University of Texas at Dallas, USA, pp.119-122.

Lofberg, J. (2006) 'Yalmip: software for solving convex (and nonconvex) optimization problems', Proceedings of American Control Conference, Minneapolis, MN, USA, pp.1-4.

Mal-Sarkar, S., Sikder, I.U. and Yu, C.S. (2009) 'Uncertainty-aware wireless sensor networks', International Journal of Mobile Communications, Vol. 7, No. 3, pp.330-345.

Mitola III, J. and Maguire, G.Q. (1999) 'Cognitive radio: making software radios more personal', IEEE Personal Communications Magazine, Vol. 6, No. 4, pp.13-18.

Needell, D. and Vershynin, R. (2009) 'Uniform uncertainty principle and signal recovery via regularized orthogonal matching pursuit', Foundations of Computational Mathematics, Vol. 9, No. 3, pp.1615-3383.

Oppenheim, V. and Shaffer, R.W. (1989) Discrete-Time Signal Processing, Prentice-Hall, New Jersey, Englewood Cliffs.

Polo, Y.L., Wang, Y., Pandharipande, W. and Leus, G. (2009) 'Compressive wideband spectrum sensing', International Conference on Acoustics, Speech, and Signal Processing 2009 (ICASSP 2009), Taipei, Taiwan, pp.2337-2340.

Sahai, A. and Cabric, D. (2005) 'Spectrum sensing - fundamental limits and practical challenges', A tutorial presented at IEEE DySpan Conference, Baltimore, pp.1-138.

Sturm, J. (1999) 'Using sedumi 1.02, a matlab toolbox for optimization over symmetric cones', Optimization Methods and Software, Vol. 11, No. 12, pp.625-653.

Tian, Z. (2008) 'Compressed wideband sensing in cooperative cognitive radio networks', Proceedings of IEEE Globecom Conference 2008, New Orleans, pp.1-5. 
Tian, Z. and Giannakis, G.B. (2006) 'A wavelet approach to wideband spectrum sensing for cognitive radios', Proceeding of 1st International Conference on Cognitive Radio Oriented Wireless Networks \& Communications (CROWNCOM), Mykonos Island, Greece, pp.1-5.

Tian, Z. and Giannakis, G.B. (2007) 'Compressed sensing for wideband cognitive radios', International Conference on Acoustics, Speech, and Signal Processing 2007 (ICASSP 2007), Honolulu, Hawaii, USA, Vol. 4, pp. IV-1357-IV-1360.

Tian, Z., Blasch, E., Li, W., Chen, G. and Li, X. (2008) 'Performance evaluation of distributed compressed wideband sensing for cognitive radio networks', Proceedings of the ISIF/IEEE International Conference on Information Fusion (FUSION), Cologne, Germany, pp.1-8.

Tibshirani, R. (1996) 'Regression shrinkage and selection via the lasso', Journal of the Royal Statistical Society, Series B (Statistical Methodology), Vol. 58, No. 1, pp.267-288.

Wang, Y., Pandharipande, A., Polo, Y.L. and Leus, G. (2009) 'Distributed compressive wide-band spectrum sensing', IEEE Proceedings of Information Theory Application (ITA 09), San Diego, CA, pp. $1-4$

Yucek, T. (2007) Channel, Spectrum, and Waveform Awareness in OFDM-Based Cognitive Radio Systems, PhD Dissertation, University of South Florida, Florida, USA.

Yucek, T. and Arslan, H. (2009) 'A survey of spectrum sensing algorithms for cognitive radio applications', IEEE Communications Surveys \& Tutorials, Vol. 11, No. 1, pp.116-130.

Zeng, F., Li, C. and Tian, Z. (2011) 'Distributed compressive spectrum sensing in cooperative multi-hop wideband cognitive networks', IEEE Journal of Selected Topics in Signal Processing, Vol. 5, No. 1, pp.37-48. 